\title{
1 Title: Reliance on polyfunctional tissue leads to a reproduction-immunity tradeoff due to
}

2 inherent constraint

3

4 Authors:

5 Vanika Gupta ${ }^{1,2 \star}$, Ashley M. Frank ${ }^{1}$, Nick Matolka ${ }^{1}$, Brian P. Lazzaro ${ }^{1,2 \star}$

6

$7 \quad{ }^{1}$ Department of Entomology, Cornell University, Ithaca, New York, USA

$8{ }^{2}$ Cornell Institute of Host-Microbe Interactions and Disease, Cornell University, Ithaca, New

9 York, USA

*Correspondence to: VG: vg272@cornell.edu, BPL: bplazzaro@cornell.edu

\section{Abstract:}

The use of one tissue for multiple purposes can result in constraints, impaired function, and tradeoffs. The insect fat body performs remarkably diverse functions including metabolic control, reproductive provisioning, and systemic immune responses. Immunity and reproduction are observed to trade off in many organisms, although the mechanistic basis for the tradeoff is generally unknown. More generally, how do polyfunctional tissues simultaneously execute multiple distinct physiological functions? Using single-nucleus sequencing, we determined the Drosophila melanogaster fat body executes diverse basal functions with heterogenous cellular subpopulations. However, as an emergency function, the immune response engages the entire tissue. We found that reproductively active females exhibit impaired capacity to produce new protein in response to infection, resulting in the reproduction-immunity tradeoff. We suggest that such inherent internal limitations may provide a general explanation for the wide prevalence of physiological and evolutionary tradeoffs.

\section{Introduction}

The need to balance multiple physiologically demanding and resource-intensive processes limits the ability of an organism to maximize performance in any one area. When two or more processes depend on a single tissue or resource pool, they unavoidably constrain each

32 other, resulting in tradeoffs between the associated traits. Such tradeoffs are central to life 33 history theory and affect the health, fitness, and evolution of all living organisms (1-3). 
Reproduction and immunity are two traits that trade off with each other across a broad diversity of systems $(4,5)$ but the mechanisms and physiological constraints that underlie this tradeoff are poorly understood. In Drosophila melanogaster females, mating results in a rapid, endocrinologically-mediated drop in resistance to bacterial infection (6). We hypothesized that this tradeoff arises due to physiological constraints of using the same tissue, the abdominal fat body, for both reproductive investment and systemic immunity, and that understanding the basis for this tradeoff could serve as a model for understanding constraints on polyfunctional tissues in general.

The insect fat body is a highly multifunctional tissue that is engaged in central metabolic regulation, nutrient storage, detoxification of xenobiotics, reproductive egg provisioning, and mounting of systemic immune responses (7). Thus, this single tissue performs the functions of several vertebrate organs. The fat body is remarkably dynamic. For example, a bacterial infection significantly changes the expression of several hundred genes in the fat body of Drosophila melanogaster, including as much as 1000-fold induction of genes encoding antimicrobial peptides and marked down-regulation of glycolytic and basal metabolic pathways (8-10). Upon mating and sperm storage, the same tissue significantly upregulates genes involved in egg provisioning as the females increase their investment egg production (7). Reproduction and immune responses are both energetically demanding (11) and a female may need to simultaneously execute these processes as well as others. Given the finite number of cells and limited capacity for transcription and translation within each cell, how does one tissue achieve so many functions at once? Is the tissue composed of specialized subpopulations of cells that are individually devoted to each function? Or do all cells of the tissue perform all functions to a limited degree? When the tissue responds to stimulus, do the identities or sizes of cellular subpopulations change, or does each cell of the tissue alter its transcriptional profile in concert? Does the simultaneous execution of multiple processes by the single tissue constrain performance of each process?

To begin address these questions, we performed single-nucleus RNA sequencing (snRNAseq) on the fat bodies of $D$. melanogaster females in a replicated factorial design combining mating and bacterial infection. Mature adult female $D$. melanogaster were either mated in order to activate reproductive investment (M_) or held as virgin to limit reproductive investment ( $\left.\mathrm{V}_{-}\right)$, and were either given a systemic bacterial infection with Providencia rettgeri to stimulate an immune response $(\mathrm{I})$ or were held uninfected ( $U$ ). We observed significantly 
post-infection ( $p=0.0001$; Fig. $1 \mathrm{~A}$ ), in accordance with previous observations $(12,13)$ and demonstrating the expected tradeoff. We repeated each factorial treatment (VU, VI, MU, MI) in two independent biological replicates to generate a total of 8 samples for snRNAseq. From each sample, we dissected and pooled fat bodies from the abdomens of 40 female flies. The gut and ovaries are easily removed from the fat body tissue, but other cell types such as oenocytes, muscle cells, and hemocytes are harder to separate from fat body tissues and thus are coisolated. We purified individual nuclei from the pooled tissues using a Dounce homogenizer followed by centrifugation onto a sucrose cushion (14). We performed snRNAseq using the 10X Genomics Chromium platform, loading at least 7000 nuclei per sample and sequencing at least 16,000 reads per nucleus for a minimum of 112 million reads per sample.

We identified 19 expression clusters representing distinct cellular subpopulations (Fig. 1B, Fig. S1, S2) with $90 \%$ of the nuclei present in the eleven most abundant clusters (Fig. 1C). We assigned putative functional identities to each cluster based on the significantly high expression ( $p$-adj <0.01) of diagnostic marker genes. Expression of top marker genes for the first eleven clusters is shown in Fig. 1D, and a full list of key expressed genes can be found in Table S1 and is illustrated in Fig. S3. The Supplementary Online Material contains detailed descriptions of the expression patterns and inferred functions for each cluster. We found significantly high expression of marker genes that are conventionally associated with fat body in six major clusters: $0,1,2,5,7$ and 10 . These six clusters contain approximately $60 \%$ of all the nuclei sequenced and demonstrate that the fat body tissue is composed of heterogeneous cell subtypes. An additional $5 \%$ of nuclei map to low-abundance fat body clusters (Clusters 11, 12, 13, and 16). Clusters 0 and 2 were defined by high expression of yolk proteins 1 and 3 (Fig. 1D), while cluster 1, 5, 7 and 10 respectively had high expression of deadhead, megalin, oskar, and vitelline membrane 26Ab. All these marker genes are associated with oogenesis and egg development. Remarkably, the relative size of these clusters did not change significantly across the four treatment groups (Fig. 1C), indicating that the fat body does not respond to mating or infection by shifting the proportional representation of these specific cellular subpopulations. We infer that the remaining $35 \%$ of nuclei do not come from fat body tissue. Based on previously well-characterized cell-specific transcriptional markers, we determined that Cluster 4 is muscle (7\% of sequenced nuclei), Cluster 6 is oenocytes (7\%), Cluster 8 is hemocytes $(5 \%)$, and Cluster 9 is uncharacterized (2\%). Cluster $3(10 \%$ of sequenced nuclei) remains uncharacterized but shows properties similar to both fat body and pericardial cells (see detailed 
description in Supplement, Fig. S4). These tissues are physically contiguous with the fat body, and interact with and have partially overlapping functions with the fat body tissue $(15,16)$.

Upon mating, D. melanogaster females store sperm and begin to lay fertilized eggs, which requires increased investment in oogenesis (17). We asked whether the investment in reproduction varied across the six distinct subpopulations of the fat body tissue by clusterspecific differential gene expression analysis. When comparing virgin and mated females (24 hours post-mating) in the absence of infection, we found 186 differentially expressed genes across the six clusters with 145 genes significantly upregulated and 41 genes significantly downregulated (FDR <0.01; Table S2). We observed that none of the 186 genes were differentially regulated across all the six subpopulations (Fig. S5A) while $123(66 \%)$ of these genes were differentially regulated in only one of the six subpopulations (Fig. S5A). For example, egg provisioning genes yp1 and yp3 were upregulated across four different clusters (Fig. S6A) while yp2 was upregulated in only one cluster (Table S2). This indicates that the response to and investment in mating is heterogenous across fat body subpopulations. GO enrichment analysis of differentially regulated genes in each of the six subpopulations showed enrichment for diverse functions (Table S3). Upregulated genes in both Clusters 0 and 1 were enriched for one-carbon metabolism but mediated by two different mechanisms: s-adenosyl methionine (SAM; Cluster 0) and folate (Cluster 1). Cluster 1 also showed enriched upregulation of genes encoding ribosomal proteins, which were downregulated in Cluster 2. Upregulated genes in Cluster 2 showed enrichment for amino acid biosynthesis. We identified metabolic and detoxification pathways enriched in genes upregulated in Cluster 5, and upregulated genes in both Clusters 7 and 10 were related to phospholipase A1 activity. Therefore, while all six fat body subpopulations respond to mating stimulus, their heterogeneous response suggests subfunctionalization of the cellular populations. 

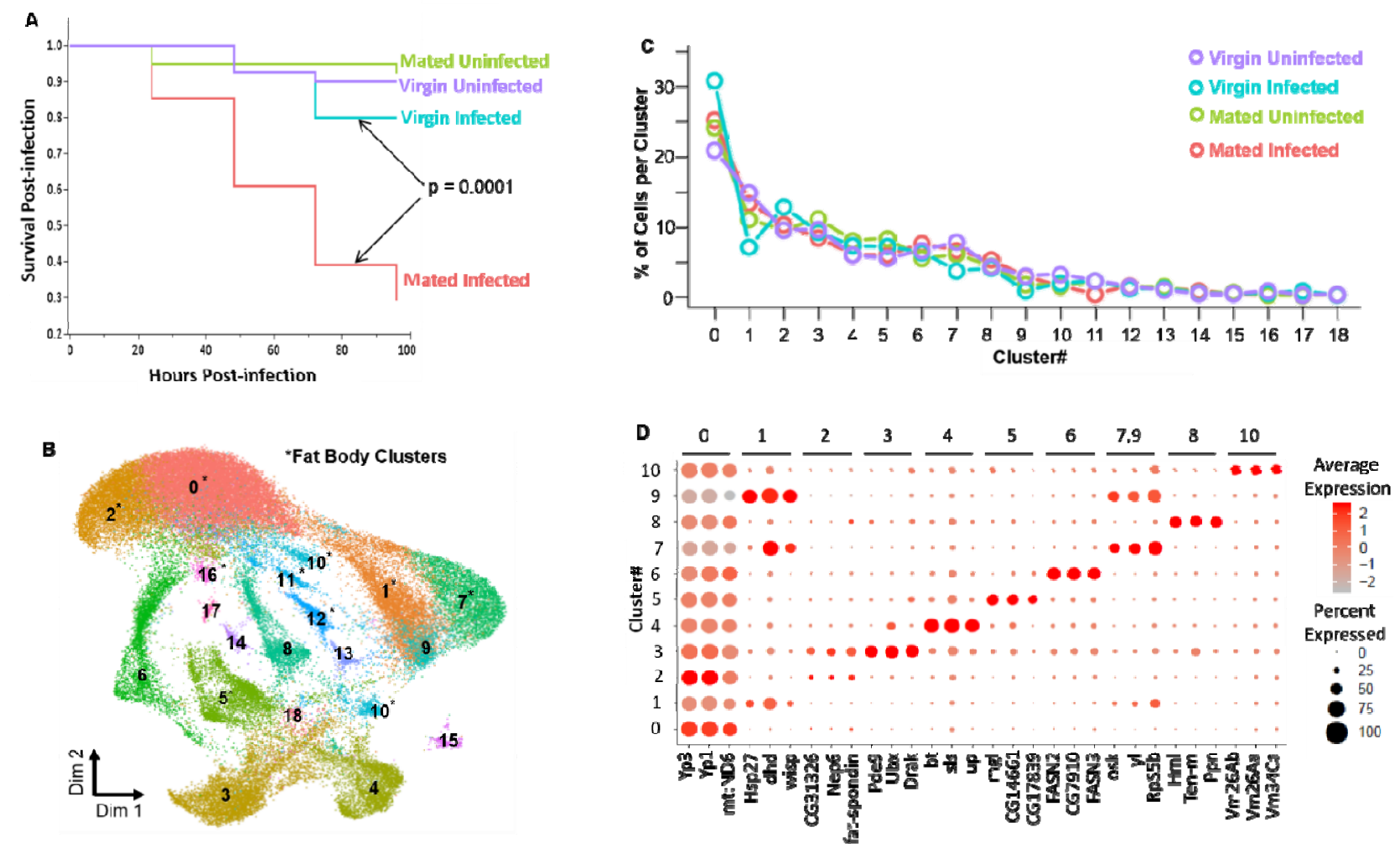

Fig. 1. Single-nucleus sequencing of Drosophila fat body tissue

(A) Cox proportional hazard analysis showed that the mated Drosophila melanogaster females have significantly lower survival than virgin females $(n=40 ; p=0.0001)$ after infection with the Gram-negative bacterium Providencia rettgeri. Survival of uninfected virgin and mated females was not different over four days. (B) Combined Uniform Manifold Approximation and Projection (UMAP) of 56,000 nuclei from two replicates each of Virgin Uninfected, Virgin Infected, Mated Uninfected, and Mated Infected colored by their treatment identity. Clusters 0,1,2,5,7, and 10 marked with asterisk $\left(^{*}\right)$ represent subpopulations of the fat body tissue. (C) Percentage distribution of nuclei from four treatments (irgin Uninfected, Virgin Infected, Mated Uninfected, and Mated Infected) across 19 clusters. All clusters are present in constant proportion across all four treatments. (D) Dot Plot showing expression of marker genes per cluster for top eleven clusters. Average expression of a marker gene in a cluster is represented by gradient of the colored dot and dot size represents the cell percentage per cluster expressing the marker. so we asked whether the whole tissue is engaged in that response or whether it maps to a restricted set of subpopulations. The answer, interestingly, is both. All clusters showed 
144

145

146

147

148

149

150

151

152

153

154

155

156

157

158

159

160

161

162

163

164

165

166

167

168

169

170

171

172

173

174

175

176

genes that encode secreted antimicrobial peptides (Figs.S6B, S6C). However, the precise expression patterns were heterogeneous after infection, with particular combinations of immune genes induced most strongly in different subsets of clusters. Across the six major fat body subpopulations, 47 genes were induced by infection in both virgin and mated females. However, twice as many genes showed significant induction after infection in virgin females than in mated females (124 versus 63, FDR <0.01; Table S4, S5), indicating a negative impact of mating on the transcriptional response to infection. We found three genes (attacin A, CG42807, and CG14322) to be upregulated across all six fat body subpopulations in virgins (Table S4, Fig. S5B) while no genes were upregulated across every subpopulation in mated females (Table S5, Fig. S5C). Around $11 \%$ genes were upregulated in 4 or more of the six subpopulations in virgins compared to $6 \%$ in mated females. To understand the functional heterogeneity of the genes expressed in each cluster, we performed cluster-specific GO enrichment analysis of the genes that are differently expressed after infection in mated and virgin females separately (Table S6). Protein processing and secretion was a significantly enriched function of upregulated genes in Clusters 0 and 2 in both virgin and mated flies. Cluster 2 in virgin infected females also showed enrichment of genes for phagosome formation (Table S6). Downregulation of ribosome constituents was observed in Cluster 1 of mated flies and Cluster 2 of virgin females. We observed that $54 \%$ of differentially expressed genes in virgins and $65 \%$ of differentially expressed genes in mated were differentially regulated in only one of the six subpopulations (Figs.S5B, S5C). These data reveal heterogeneity in infection response across the fat body and demonstrate that the tissue-wide transcriptional response to infection is dampened by mating, probably contributing to the tradeoff between reproduction and immunity.

Most of the mating- and infection- induced transcriptional changes were heavily driven by clusters 0,1 , and 2 (Table S7), representing $\sim 70 \%$ of all the nuclei from the six fat body subpopulations. We hypothesized that the involvement of such a large majority of fat body cells in resource-intensive physiological functions might constrain resource allocation, which could be reflected in coordinated regulation of gene expression networks or modules. To identify these modules, we constructed pseudotime trajectories from all the four treatments with Monocle (1921 ), representing the transition of cells between differential functional states in response to mating or infection. An initial analysis revealed that the infected and uninfected fat body cells resolved into two completely disjointed trajectories defined by infection status. Trajectory 1 contained a majority of nuclei from $\mathrm{VU}$ and $\mathrm{MU}$ treatments while Trajectory 2 contained a majority of nuclei from VI and MI (Fig. 2A, Fig. S7). This suggests that fat body cells rapidly and 
177 dramatically change expression profile upon infection with no intermediate states visible at the

178 6-hour post-infection sampling time point. Only fat body cells (inferred using Seurat-based

179 cluster analysis) were present in both of these trajectories (Fig. 2B). Other co-isolated cell types

180 were present in only one of the two trajectories; indicating that they are not strongly

181 transcriptionally responsive to infection. Using Louvain clustering in the two trajectories, we

182 identified several modules of co-regulated genes that were enriched for specific functional

183 ontologies. In Trajectory 1, we identified a module (Module 13, Fig. 2C, Table S8) with low

184 aggregate expression score that was enriched in ribosome biogenesis (Fig. S8) in mated

185 uninfected nuclei (MU) relative to virgin uninfected nuclei (VU), including CAP-dependent

186 translation initiation factors. Surprisingly, the same set of genes (Module 16, Fig. 2C, Table S9)

187 with the addition of one gene (O-fucosyltransferase 2) had a low aggregate expression score in

188 mated infected (MI) nuclei contrasted to virgin infected nuclei (VI) in Trajectory 2 (Module 16).

189 This suggests that protein synthesis might be reduced in the fat body after mating due to

190 suppressed ribosome biogenesis. Furthermore, a subset of MI nuclei showed high expression of

191 a module enriched in protein folding and degradation (Fig. S9), including genes involved in ER

192 stress and unfolded protein response (UPR; Table S10). Electron microscopy confirmed dilated

193 ER membranes in Ml fat bodies (Fig. 3), indicative of ER stress (22). Since alleviation of ER

194 stress is often attained via suppression of ribosome biogenesis to limit protein synthesis in the

195 cell $(22,23)$, a key factor underlying the observed reproduction-immunity tradeoff could be

196 reduced capacity to produce immune-related proteins in mated females due to reduced protein

197 synthesis. 

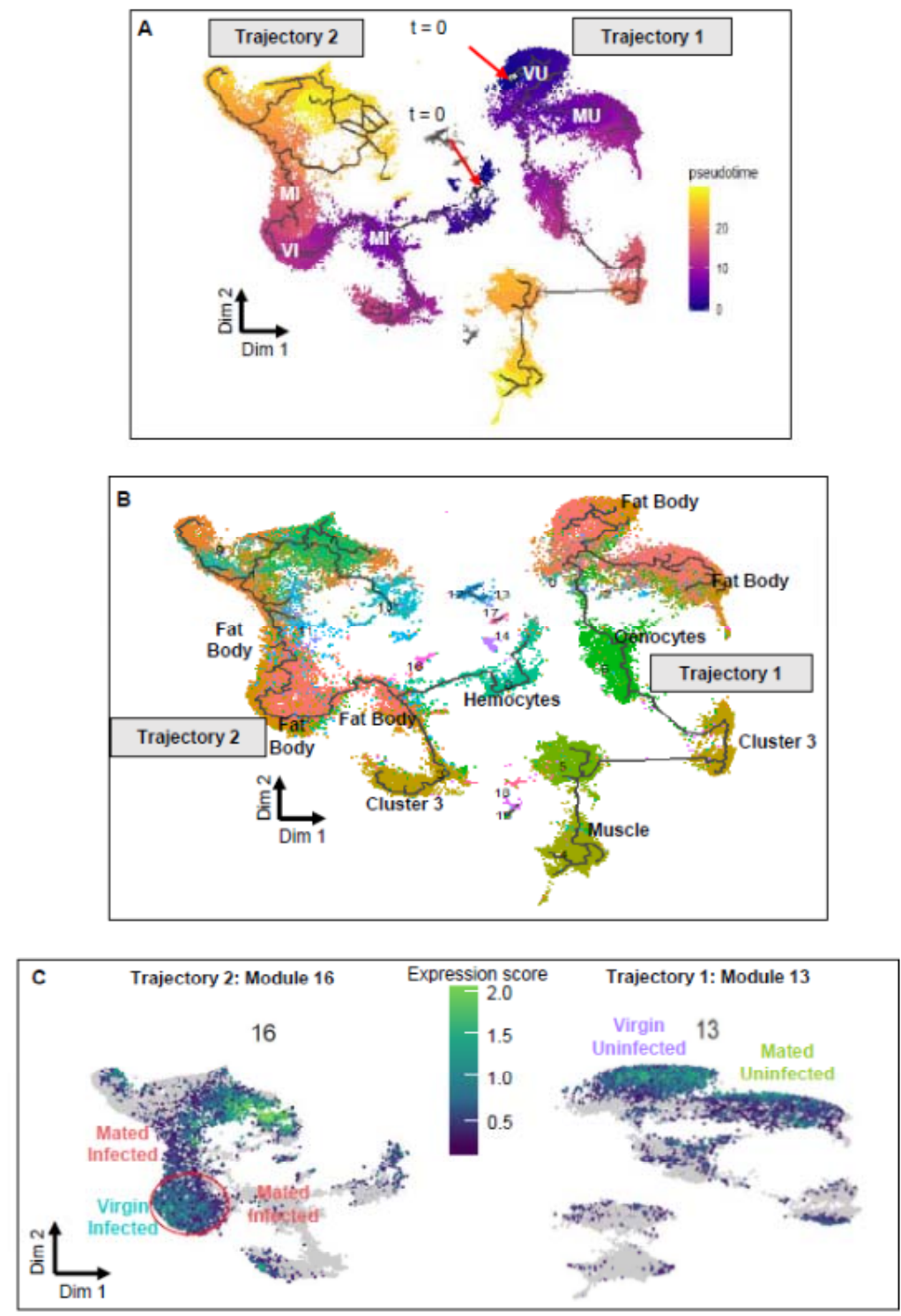

Fig. 2 Pseudotime analysis showing differentially expressed gene modules

(A) Pseudotemporal ordering of nuclei along the two trajectories calculated from trajectoryspecific $(\mathrm{t}=0)$ points. Nuclei from four treatments (irgin Uninfected $(\mathrm{VU})$, irgin Infected $(\mathrm{VI})$,

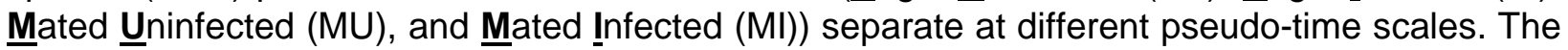
trajectories from infected nuclei are completely disjoint from the trajectories of uninfected nuclei, revealing a rapid and dramatic response to infection. (B) Monocle-based trajectory analysis separated nuclei along the two trajectories; colored by their cluster identity (from Figure 1B) show that only Fat Body nuclei are present in both trajectories. Other cell types such as oenocytes and muscle cells are present in Trajectory 1 and hemocytes are present in Trajectory 2 , indicating these cell types do not have a strong transcriptional response to infection. (C) UMAP of Module 13 (Trajectory 1) and Module 16 (Trajectory 2) showing low gene aggregate expression scores for Mated Uninfected (Trajectory 1) and Mated Infected (Trajectory 2) compared to Virgin Uninfected and Virgin Infected respectively. Gradient of color represents the aggregate expression score with bright color indicating higher aggregate expression score. 
Each dot represents a single nucleus. GO term analysis showed enrichment for ribosome biogenesis in the two modules (Tables S8, S9, Fig. S8).

217

218

219

220

221

222

223

224

225

226

227

228

229

230

231

232

233

234

235

236

237

238

\section{Virgin Infected}

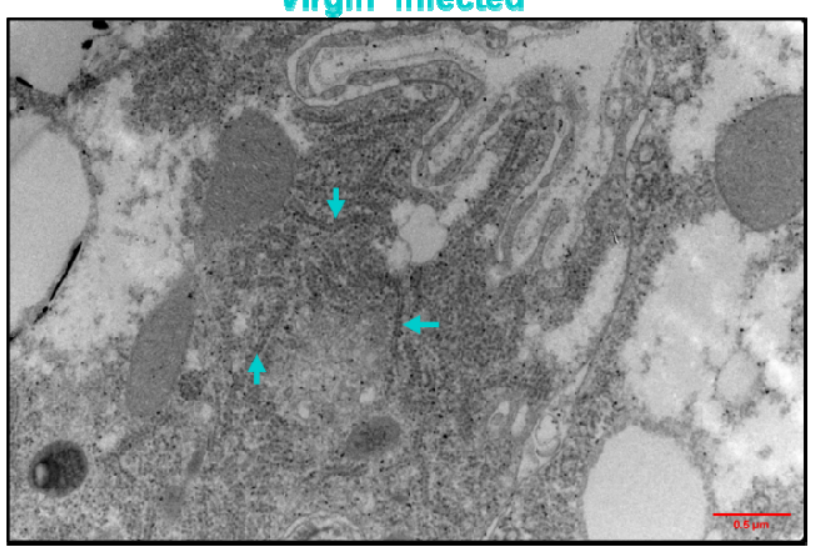

Mated Infected

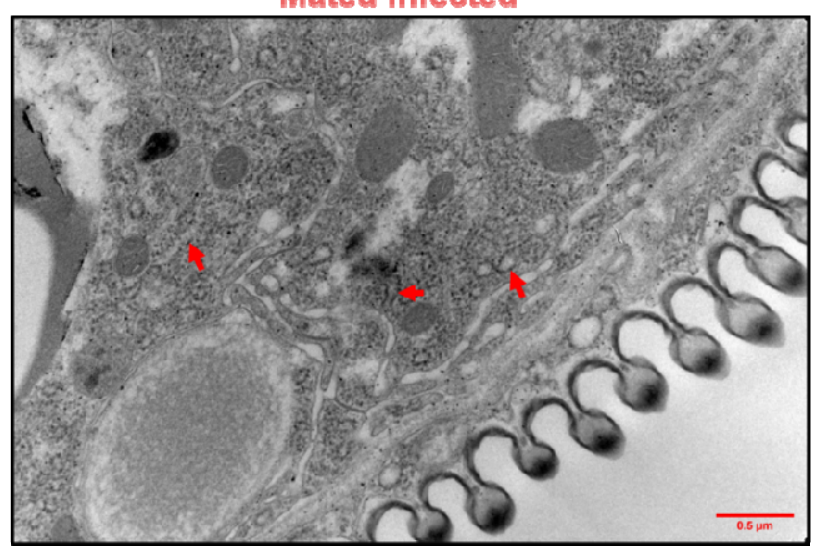

Fig. 3 Electron micrographs of endoplasmic reticulum in the fat body (ER)

Representative image ( $n=4-5$ images per treatment) showing dilation of ER membrane indicative of ER stress (right panel, red arrows) observed in fat body cells from Mated Infected females. Blue arrows show constricted ER membranes in Virgin Infected samples.

To test our hypothesis that mated infected (MI) females may lack sufficient capacity for translation in support of a full immune response to infection, we measured global protein synthesis in fat body tissues representing each of the four different treatments. We re-generated new female flies from each of the four factorial mating and infection treatments, dissected their fat bodies, and applied puromycin incorporation to label nascent polypeptides. Incorporated puromycin was then quantified on Western blots $(20,21)$. We observed significant variability in global synthesis rates across the four treatments (one-way ANOVA, $p=0.02$, Table S11) with a spike in protein synthesis after infection in virgin females $(\mathrm{VI})($ Mean $=2.2$, S.D. $=0.69)$ that fails to occur in mated (MI) females (Mean =0.8, S.D. =0.61) (Tukey's HSD, p =0.0005, Fig. 4A, $4 \mathrm{~B})$. These data are consistent with the reduction in ribosome biogenesis inferred from the sequencing data and with the hypothesis that the fat bodies of Ml females are deficient in translation capacity. As the rapidity of an induced immune response is a critical determinant of infection outcome $(22,23)$, a quantitative reduction or delay in the translation of immune response proteins such as antimicrobial peptides could contribute to the observed increased risk of death from infection in mated females. 
Since we observed a reduction in protein synthesis in mated infected (MI) flies compared to all other groups, and especially compared to virgin infected (VI) females (Fig. 4B), we hypothesized that the high demand of producing reproduction-related proteins in mated females

242 reduced capacity to translate new proteins in response to infection, even leading to ER stress in

243 MI females. We predicted that the reproduction-immunity tradeoff could be alleviated if 244 translational investment in reproductive proteins was reduced. To test this hypothesis, we mated 245 females and then placed them on food containing cycloheximide ( $\mathrm{CHX}$ ) for 18 hours. $\mathrm{CHX}$ 246 reversibly suppresses production of proteins in eukaryotes such as Drosophila (24). We 247 subsequently transferred flies to food without $\mathrm{CHX}$ for six hours to allow them to clear the drug, and then gave them bacterial infections. Females that were treated with $\mathrm{CHX}$ after mating survived infection significantly better than mated females that were not treated with $\mathrm{CHX}$ $(p<0.0001$, Fig. 4C). We also observed an increase in post-infection protein synthesis in mated

251 females pre-treated with $\mathrm{CHX}$ compared to non-treated females at six hours after infection (t (4) $252=3.63, \mathrm{p}=0.02$, Fig. 4D, Table S12). Additional experiments confirmed that $\mathrm{CHX}$ has no direct role in the survival of infection (Fig. S10, see Supplemental Material for experimental details).

254 Therefore, the observed tradeoff between reproduction and immunity is determined to be due to limited capacity for immune-related protein synthesis as a consequence of prior reproductive investment.
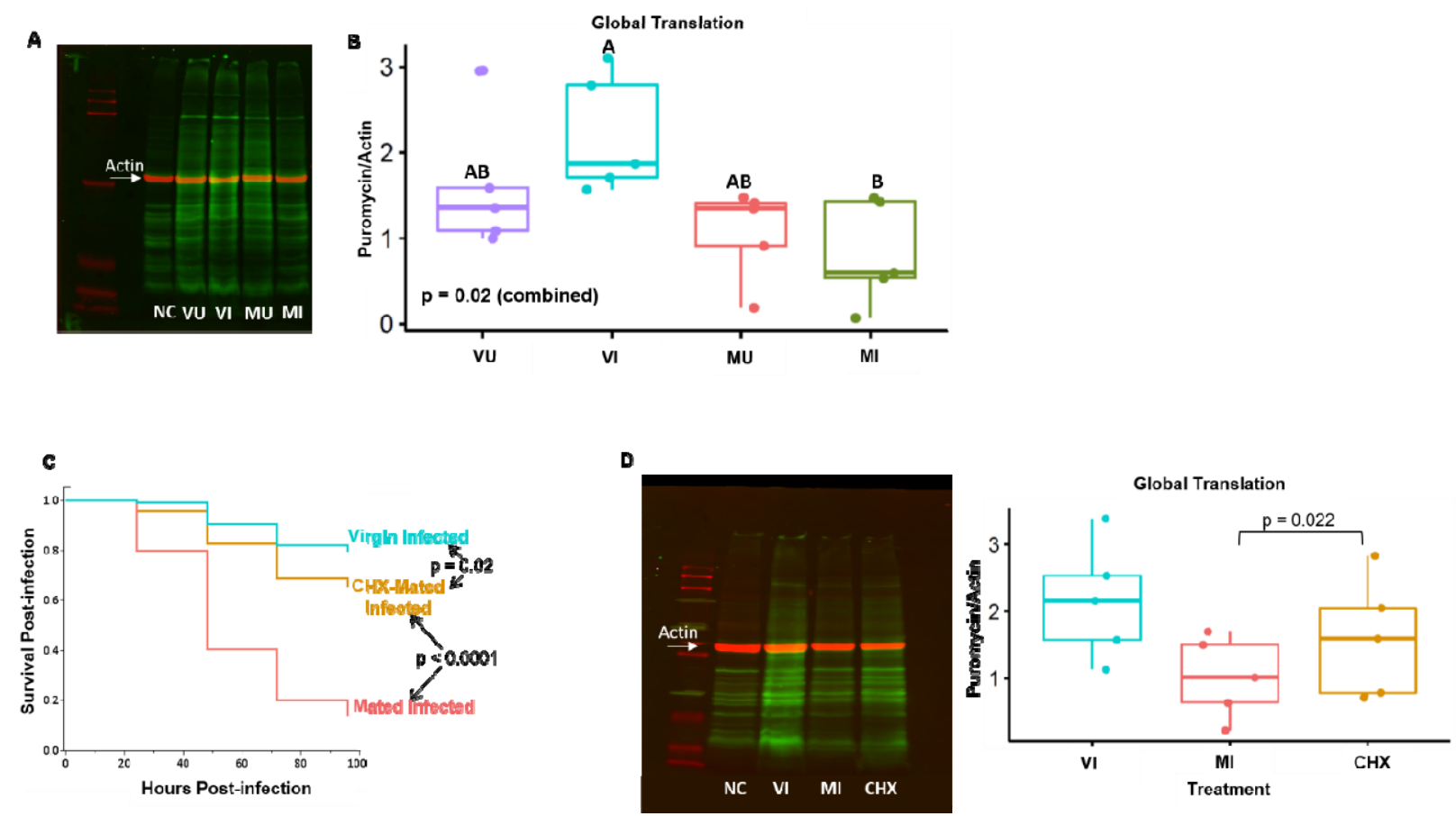
(A) Representative image of puromycin incorporation in nascent polypeptides of the fat body tissue detected using anti-puromycin antibody and Western Blotting. Secondary antibodies labelled with different fluorophores detected puromycin (Green, 800nm) and Actin (Red, 700 $\mathrm{nm}$ ). The fat bodies from Mated Infected (MI) produce noticeably less protein than those of the other treatments. Virgin Uninfected (VU), Virgin Infected (VI), Mated Uninfected (MU), and Mated Infected (MI) represent the four treatments. Negative Control (NC) (Figure A and D) shows proteins from fat body tissues which were not incubated with puromycin. (B) Quantification of relative protein synthesis using puromycin incorporation from four treatments (VU, VI, MU, and MI) ( $\mathrm{n}=5,10$ flies per treatment). Treatments not connected by same letter are significantly different (Tukey's HSD, $p<0.005$ ). Virgin Infected females synthesize significantly more protein than Mated Infected females. (C) Cox proportional hazard analysis showing rescued post-infection survival $(p<0.0001)$ of cycloheximide $(\mathrm{CHX})$ pre-treated mated females (CHX- Mated Infected) compared to non-treated Mated Infected ( $\mathrm{n}=35-40$ flies per treatment per replicate, three independent replicates). (D) Representative Western Blot image of puromycin incorporation from $\mathrm{CHX}$ pre-treatment of mated females $(\mathrm{CHX})$. Results suggest $\mathrm{CHX}$ treatment partially rescues protein synthesis in response to infection compared to nontreated mated females (MI) (Paired Student's t-test, $\mathrm{p}=0.02$ ). Puromycin incorporation was measured after six hours of infection.

The impaired ability of the fat body to synthesize proteins in response to infection while simultaneously investing in reproduction illustrates a tradeoff driven by physiological constraint. The fact that immunity can be partially rescued with $\mathrm{CHX}$ treatment suggests the potential for plasticity in this tradeoff. Flies could, in theory, sustain greater immune capacity by reducing their commitment to reproductive investment. Thus, genetic variation for reproductive investment could allow evolutionary adaptation to infection risk. Reduced translation specifically in the fat body (25) extends lifespan in flies (26) through evolutionarily conserved mechanisms (27) observed across other organisms such as $C$. elegans $(28,29)$ and mouse $(30)$, whereas mating and reproduction are costly and negatively affects lifespan in fruit flies and other organisms (31). Translation in the fat body could therefore additionally be a mechanism mediating reproduction-longevity tradeoff. It seems likely that environmental factors, such as amino acid nutrition, may also influence the shape of these tradeoffs.

Managing competing physiological demands is a critical challenge for any polyfunctional tissue. We find here that the Drosophila melanogaster fat body executes diverse basal functions via heterogeneous cellular subpopulations. However, the whole tissue becomes engaged in an immune response. The gene expression markers that we have identified as defining the cellular subpopulations can serve to develop tags for future research into the dynamism and spatial structure of the Drosophila fat body. The fat body is a remarkable tissue that is highly responsive in regulating multiple aspects of physiology. However, while the fat body is enormously flexible, the shared reliance of multiple functions on a single tissue will inherently 
lead to constraints and tradeoffs. As we have shown in defining a reproduction-immunity tradeoff, compound stresses can overwhelm the tissue and lead to adverse outcomes. Understanding strategies that polyfunctional tissues use for balancing critical functions at the whole-tissue and sub-tissue levels can elucidate general mechanisms of physiological and evolutionary tradeoffs that underpin life history theory.

\section{References}

1. Roff DA. Life history evolution . Sinauer Associates; 2002.527 p. Doi: https://global.oup.com/ushe/product/life-history-evolution$9780878937561 ? \mathrm{cc}=$ us\&lang=en\&

2. Stearns S. The Evolution of Life Histories. OUP Oxford; 1992.

3. Sheldon BC, Verhulst S. Ecological immunology: costly parasite defences and trade-offs in evolutionary ecology. Trends Ecol Evol . 1996 Aug ;11 (8):317-21. Doi: http://www.ncbi.nlm.nih.gov/pubmed/21237861

4. Schwenke RA, Lazzaro BP, Wolfner MF. Reproduction-Immunity Trade-Offs in Insects. Annu Rev Entomol . 2016 Mar 11 ;61 (1):239-56. Doi: http://www.annualreviews.org/doi/10.1146/annurev-ento-010715-023924

5. Norris K, Evans MR. Ecological immunology: Life history trade-offs and immune defense in birds. Behav Ecol. 2000;11 (1):19-26.

6. Schwenke RA, Lazzaro BP. Juvenile Hormone Suppresses Resistance to Infection in Mated Female Drosophila melanogaster. Curr Biol . 2017 ;27:1-6. Doi: http://dx.doi.org/10.1016/j.cub.2017.01.004

7. Arrese EL, Soulages JL. Insect fat body: energy, metabolism, and regulation. Annu Rev Entomol . 2010 ;55:207-25. Doi: http://www.ncbi.nlm.nih.gov/pubmed/19725772

8. Li S, Yu X, Feng Q. Fat body biology in the last decade. Annu Rev Entomol. 2019;64:315-33.

9. De Gregorio E, Spellman PT, Rubin GM, Lemaitre B. Genome-wide analysis of the Drosophila immune response by using oligonucleotide microarrays. Proc Natl Acad Sci U S A. 2001;98 (22):12590-5.

10. Clark RI, Tan SWS, Péan CB, Roostalu U, Vivancos V, Bronda K, et al. XMEF2 is an in vivo immune-metabolic switch. Cell. 2013;155 (2):435.

11. Segerstrom SC. Stress, energy, and immunity: An ecological view. Curr Dir Psychol Sci. 2007;16 (6):326-30.

12. Short SM, Wolfner MF, Lazzaro BP. Female Drosophila melanogaster suffer reduced defense against infection due to seminal fluid components. J Insect Physiol. 2012;

13. Fedorka KM, Linder JE, Winterhalter W, Promislow D. Post-mating disparity between potential and realized immune response in Drosophila melanogaster. Proc R Soc B Biol Sci. 2007;

14. Gupta V, Lazzaro BP. A robust method to isolate Drosophila fat body nuclei for transcriptomic analysis. 2021;

15. Droujinine IA, Perrimon N. Interorgan Communication Pathways in Physiology: Focus on Drosophila. Annu Rev Genet . 2016 Nov 23;50 (1):539-70. Doi: http://www.annualreviews.org/doi/10.1146/annurev-genet-121415-122024

16. Rajan A, Perrimon N. Drosophila as a Model for Interorgan Communication: Lessons from Studies on Energy Homeostasis. Dev Cell . 2011 Jul;21 (1):29-31. Doi: https://www.ncbi.nlm.nih.gov/pmc/articles/PMC3624763/pdf/nihms412728.pdf 
17. Bloch Qazi MC, Heifetz Y, Wolfner MF. The developments between gametogenesis and fertilization: Ovulation and female sperm storage in Drosophila melanogaster. Dev Biol. 2003;256 (2):195-211.

18. Dionne MS. Immune-metabolic interaction in Drosophila. Fly (Austin). 2014;8 (2):75-9.

19. Trapnell C, Cacchiarelli D, Grimsby J, Pokharel P, Li S, Morse M, et al. The dynamics and regulators of cell fate decisions are revealed by pseudotemporal ordering of single cells. Nat Biotechnol . 2014;32 (4):381-6. Doi: http://dx.doi.org/10.1038/nbt.2859

20. Qiu X, Hill A, Packer J, Lin D, Ma YA, Trapnell C. Single-cell mRNA quantification and differential analysis with Census. Nat Methods. 2017;14 (3):309-15.

21. Qiu X, Mao Q, Tang Y, Wang L, Chawla R, Pliner HA, et al. Reversed graph embedding resolves complex single-cell trajectories. Nat Methods . 2017;14 (10):979-82. Doi: http://dx.doi.org/10.1038/nmeth.4402

22. Back SH, Kaufman RJ. Endoplasmic reticulum stress and type 2 diabetes. Annu Rev Biochem. 2012;81:767-93.

23. Walter P, Ron D. The Unfolded Protein Response: From Stress Pathway to Homeostatic Regulation. Science (80- ) . 2011 Nov 25;334 (6059):1081-6. Doi:

https://pubmed.ncbi.nlm.nih.gov/22116877/

24. Bownes $M$, Scott $A$, Blair $M$. The use of an inhibitor of protein synthesis to investigate the roles of ecdysteroids and sex-determination genes on the expression of the genes encoding the drosophila yolk proteins. Development. 1987;101 (4):931-41.

25. Tain LS, Sehlke R, Jain C, Chokkalingam M, Nagaraj N, Essers P, et al. A proteomic atlas of insulin signalling reveals tissue-specific mechanisms of longevity assurance. Mol Syst Biol. 2017;13 (9):939.

26. Wang D, Cui Y, Jiang Z, Xie W. Knockdown expression of eukaryotic initiation factor 5 Cterminal domain containing protein extends lifespan in Drosophila melanogaster. Biochem Biophys Res Commun . 2014;446 (2):465-9. Doi: http://dx.doi.org/10.1016/j.bbrc.2014.02.133

27. McElwee JJ, Schuster E, Blanc E, Piper MD, Thomas JH, Patel DS, et al. Evolutionary conservation of regulated longevity assurance mechanisms. Genome Biol. 2007;8 (7).

28. Hansen M, Taubert S, Crawford D, Libina N, Lee SJ, Kenyon C. Lifespan extension by conditions that inhibit translation in Caenorhabditis elegans. Aging Cell. 2007;6 (1):95110.

29. Pan KZ, Palter JE, Rogers AN, Olsen A, Chen D, Lithgow GJ, et al. Inhibition of mRNA translation extends lifespan in Caenorhabditis elegans. Aging Cell. 2007;6 (1):111-9.

30. Thompson ACS, Bruss MD, Price JC, Khambatta CF, Holmes WE, Colangelo M, et al. Reduced in vivo hepatic proteome replacement rates but not cell proliferation rates predict maximum lifespan extension in mice. Aging Cell. 2016;15 (1):118-27.

31. Heyland TF and A. Mechanisms of Life History Evolution: The Genetics and Physiology of Life History Traits and Trade-Offs . Doi:

https://oxford.universitypressscholarship.com/view/10.1093/acprof:oso/9780199568765.0 01.0001/acprof-9780199568765

Acknowledgments: We thank Peter Schweitzer for his assistance with sequencing. John Grazul and Katherine A. Spoth assisted with sample preparation and acquisition of electron microscopy images. We thank Profs. Mariana Wolfner, Robert Reed, Nicolas Buchon, and Nilay Yapici and Garrett League, Kathleen Gordon and Radhika Ravikumar for their feedback on the manuscript. Funding: This work was funded from NIH grants R03 Al144882 and R01 Al141385. This work made use of the Cornell Center for Materials Research Shared Facilities which are supported through the NSF MRSEC program (DMR-1719875). Imaging data was acquired through the Cornell Institute of Biotechnology's Imaging Facility, with $\mathrm{NIH}$ 
bioRxiv preprint doi: https://doi.org/10.1101/2021.01.28.428655; this version posted January 29,2021 . The copyright holder for this preprint (which was not certified by peer review) is the author/funder, who has granted bioRxiv a license to display the preprint in perpetuity. It is made available under aCC-BY-NC-ND 4.0 International license.

393 1S10RR025502 funding for the shared Zeiss LSM 710 Confocal Microscope; Author 394 contributions: Conceptualization: VG, BPL; Methodology: VG; Formal analysis: VG; 395 Investigation: VG, AMF, NM; Writing: VG, BPL. Competing interests: Authors declare no 396 competing interests. 